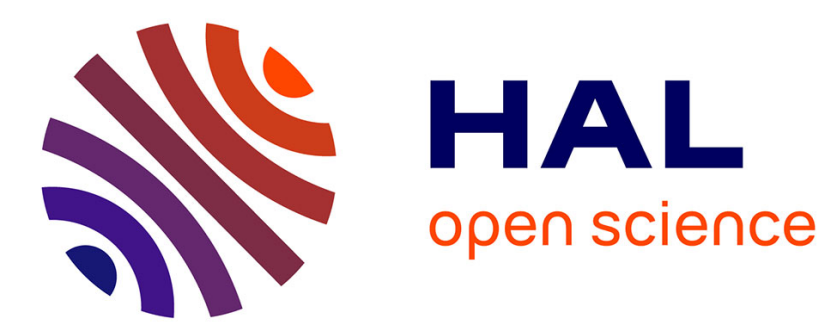

\title{
Characterization of vortical structures and loads based on time-resolved PIV for asymmetric hovering flapping flight
}

\author{
Thierry Jardin, Alain Farcy, Laurent David
}

\section{> To cite this version:}

Thierry Jardin, Alain Farcy, Laurent David. Characterization of vortical structures and loads based on time-resolved PIV for asymmetric hovering flapping flight. Experiments in Fluids, 2009, vol. 46 (nº 5), pp. 847-857. 10.1007/s00348-009-0632-7 . hal-01132123

\section{HAL Id: hal-01132123 \\ https://hal.science/hal-01132123}

Submitted on 31 Mar 2015

HAL is a multi-disciplinary open access archive for the deposit and dissemination of scientific research documents, whether they are published or not. The documents may come from teaching and research institutions in France or abroad, or from public or private research centers.
L'archive ouverte pluridisciplinaire HAL, est destinée au dépôt et à la diffusion de documents scientifiques de niveau recherche, publiés ou non, émanant des établissements d'enseignement et de recherche français ou étrangers, des laboratoires publics ou privés. 


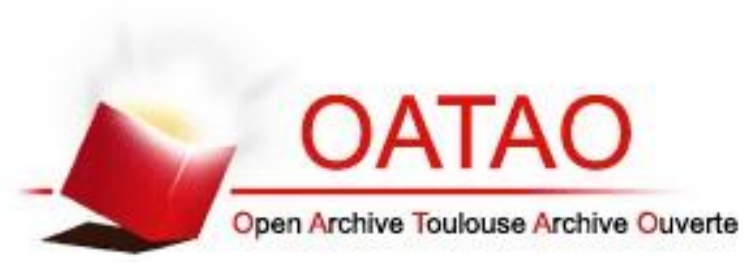

\section{Open Archive Toulouse Archive Ouverte (OATAO)}

OATAO is an open access repository that collects the work of Toulouse researchers and makes it freely available over the web where possible.

This is an author-deposited version published in: http://oatao.univ-toulouse.fr/ Eprints ID: 13654

To link to this article: DOI:10.1007/s00348-009-0632-7

URL: http://dx.doi.org/10.1007/s00348-009-0632-7

To cite this version: Jardin, Thierry and David, Laurent and Farcy, Alain Characterization of vortical structures and loads based on time-resolved PIV for asymmetric hovering flapping flight. (2009) Experiments in Fluids, vol. $46\left(\mathrm{n}^{\circ}\right.$ 5). pp. 847-857. ISSN 0723-4864

Any correspondence concerning this service should be sent to the repository administrator: staff-oatao@inp-toulouse.fr 


\title{
Characterization of vortical structures and loads based on time-resolved PIV for asymmetric hovering flapping flight
}

\author{
T. Jardin · Laurent David · A. Farcy
}

\begin{abstract}
Flight agility, resistance to gusts, capability to hover coupled with a low noise generation might have been some of the reasons why insects are among the oldest species observed in nature. Biologists and aerodynamicists focused on analyzing such flight performances for diverse purposes: understanding the essence of flapping wings aerodynamics and applying this wing concept to the development of micro-air vehicles (MAVs). In order to put into evidence the fundamentally non-linear unsteady mechanisms responsible for the amount of lift generated by a flapping wing (Dickinson et al. in Science 284:19541960, 1999), experimental and numerical studies were carried out on typical insect model wings and kinematics. On the other hand, in the recent context of MAVs development, it is of particular interest to study simplified nonbiological flapping configurations which could lead to lift and/or efficiency enhancement. In this paper, we propose a parametrical study of a NACA0012 profile undergoing asymmetric hovering flapping motions at Reynolds 1000. On the contrary to normal hovering, which has been widely studied as being the most common configuration observed in the world of insects, asymmetric hovering is characterized by an inclined stroke plane. Besides the fact that the vertical force is hence a combination of both lift and drag (Wang in J Exp Biol 207:1137-1150, 2004), the specificity of such motions resides in the vortex dynamics which present distinct behaviours, whether the upstroke angle of attack leads to a partially attached or a strong separated flow, giving more or less importance to the wake capture phenomenon. A direct consequence of the previous
\end{abstract}

T. Jardin $\cdot$ L. David $(\bowtie) \cdot$ A. Farcy

LEA, University of Poitiers, CNRS, ENSMA, SP2MI,

bd Marie et Pierre Curie, 86962 Futuroscope, France

e-mail: laurent.david@univ-poitiers.fr remarks relies on the enhancement of aerodynamic efficiency with asymmetry. If several studies reported results based on the asymmetric flapping motion of dragonfly, only few works concentrated on parametrizing asymmetric motions (e.g. Wang in Phys Rev Lett 85:2216-2219, 2000). The present study relies on TR-PIV measurements which allow determination of the vorticity fields and provide a basis to evaluate the resulting unsteady forces through the momemtum equation approach.

\section{Introduction}

The aerodynamic performances of insects, in terms of lift generation, hover or flight agility, have always fascinated biologists and aerodynamicists. Pioneer works focused on explaining such performances by means of the quasi-steady approach. Walker (1925), von Holst and Kuchemann (1941) and Osborne (1951) introduced the latter (as well as the blade element theory) to flapping flight analysis but were critically reviewed by Weis-Fogh and Jensen (1956) who denoted a lack of preciseness. Jensen (1956) measured both (1) the velocities and angles of attack characterizing the wing motion of a tethered locust by means of a highspeed camera and (2) the lift and drag polar of a dissected wing; giving an evaluation of the quasi-steady aerodynamic forces in forward flight. Later, Weis-Fogh (1973) showed that the quasi-steady approach is suitable for explaining the amount of lift generated by most species of insects but may somehow fail when considering hovering flight. This observation was evidence that unsteady mechanisms are essential in insects aerodynamics. The idea was supported by Ellington (1984) who reviewed the quasisteady studies carried out on different species in hovering 
flapping flight. As a consequence, researchers then concentrated their efforts on determining the unsteady mechanisms responsible for the generation of strong aerodynamic forces. Basically, apart from the clap-andfling mechanism (Weis-Fogh 1973) which is specific to particular species, three phenomena may be described: (1) the presence of a leading edge vortex (LEV) or dynamic stall mechanism (Walker 1931; Polhamus 1971; Maxworthy 1979; Dickinson and Götz 1993), (2) the Kramer effect analogous to the supplementary circulation generated by the combined translating and rotating motions (Kramer 1932; Bennett 1970; Dickinson et al. 1999; Sane and Dickinson 2002) and (3) the wake capture phenomenon, arising from the interaction between the wing and its own wake induced during previous strokes (Dickinson 1994; Dickinson et al. 1999; Birch and Dickinson 2003). In addition to these phenomena, one should keep in mind the concept of added mass (or virtual mass) acting as a noncirculatory reaction force due to the accelerated fluid when the wing motion is not constant.

Previous findings mainly emerged from the observation of the flow generated by three-dimensional dynamically scaled robots mimicking the flight of insects. Such configurations (i.e. revolving wings) imply the presence of a velocity gradient along the wing span which may tend to stabilize the behaviour of the leading edge vortex (Maxworthy 1979; van den Berg and Ellington 1997; Liu et al. 1998). On the other hand, two-dimensional approaches proved to be an efficient way for characterizing the flow features when considering the influence of wing kinematics.

It clearly appears that one of the inherent characteristics of unsteady phenomena relies on their dependence to wing kinematics. In order to understand the influence of the latter on the vortex dynamics, hence the generation of lift and drag forces in hovering flapping flight, parametrical numerical and experimental studies were conducted. Sane and Dickinson (2001, 2002) and Singh et al. (2005) experimentally investigated the influence of angle of attack, flip timing, stroke deviation, angular velocity and centre of rotation by means of dynamically scaled robots equipped with 2D force sensors. Similar parameters were numerically analyzed by Sun and Tang (2002) and Wu and Sun (2004). Wang et al. (2004) and Kurtulus (2005) provided a comparison of parametrical studies performed both experimentally and numerically. These previous works focused on the symmetric or "normal" hovering flapping flight configurations, known as the most common configurations in the world of insects, for which the wing translates along a horizontal stroke plane. Alternative configurations rely on the desymmetrization of the motion (e.g. different upstroke and downstroke angles of attack) resulting in an inclined stroke plane in order to maintain hovering; i.e. zero mean horizontal force. The particularity of such asymmetric motions is that the vertical force necessary to keep the insect aloft is hence a combination of both lift and drag (Wang 2004). Several studies reported results based on the flight of the dragonfly (Somps and Luttges 1985; Gustafson and Leben 1991; Wang 2000; Sun and Lan 2004; Thomas et al. 2004).

Recently, the concept of flapping wings appeared as a possible alternative to the conventional fixed and rotary wings in the development of micro air-vehicles (MAVs). This new generation of unmanned aircraft is characterized by a maximum dimension of $15 \mathrm{~cm}$, which places the corresponding aerodynamic flows in the range of low Reynolds numbers $\left(10^{2}-10^{4}\right)$, comparable to the ones found in insect flight. Thus, in order to optimize such flying devices, it is of particular interest to study simplified nonbiological flapping motions which could lead to lift and/or efficiency enhancement. Platzer and Jones (2006) proposes a review of the recent works dedicated to the development of MAVs. The questions of flapping modes, amplitudes, frequencies for optimum cruise flight as well as hovering flight or wing interactions are addressed, giving rise to the authors' prototype.

In this paper, we present an experimental parametrical study of asymmetric hovering flapping motions, which, to our knowledge, has not been yet proposed in the literature. Time Resolved Particle Image Velocimetry (TR-PIV) is performed on a dynamically scaled NACA0012 profile at Reynolds 1000. The resulting flow fields allow both (1) the analysis of the spatial and temporal behaviour of the wake structure and (2) the evaluation of correlated unsteady forces determined by means of the momentum equation approach (Noca et al. 1997; Unal et al. 1997). The comparison with symmetric hovering flapping motions (Kurtulus et al. 2008) reveals significant different vortex dynamics principally arising from the absence of significant wake capture during downstroke when the upstroke angle of attack is set to a low value (e.g. $20^{\circ}$ ).

\section{Experimental tools}

\subsection{Wing kinematics}

The wing kinematics result from the combination of translating and rotating motions as shown in Fig. 1. Basically, the flapping motion may be separated into different phases whether the wing is translating at constant speed and fixed angle of attack (region T) or is subjected to both varying translation speed and rotating motion (regions $\mathrm{R}$ ). The rotation is applied around a spanwise axis located $1 / 4$ chord away from the leading edge. Region $\mathrm{T}$ and regions $\mathrm{R}$ are, respectively 4- and 1-chord long, so that the wing travels along a total course of 6 chords during one stroke. 
Fig. 1 Description of the flapping motion

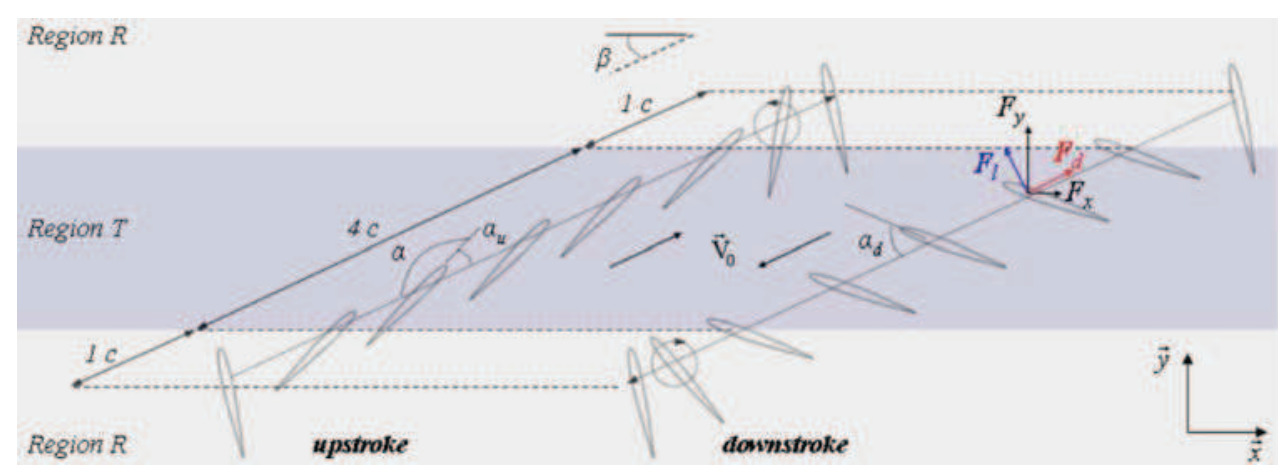

$T=\frac{4 c}{\left|V_{0}\right|}\left(\frac{\pi}{2}+2\right)$

The constant wing velocity $V_{0}$ reached during the pure translation phases (region $\mathrm{T}$ ) is calculated with respect to the Reynolds number such that:

$R e=\frac{c V_{0}}{V}$

where $c$ is the chord of the NACA0012 profile and $v$ the kinematic viscosity. The parametrical study relies on the corresponding downstroke and upstroke angles of attack $\alpha_{\mathrm{d}}$ and $\alpha_{\mathrm{u}}$. A total of 9 configurations are investigated, $\alpha_{\mathrm{d}}$ and $\alpha_{\mathrm{u}}$ being set as couples to $\left(30^{\circ}, 10^{\circ}\right),\left(30^{\circ}, 20^{\circ}\right),\left(30^{\circ}, 30^{\circ}\right)$, $\left(45^{\circ}, 20^{\circ}\right),\left(45^{\circ}, 30^{\circ}\right),\left(45^{\circ}, 45^{\circ}\right)$ and $\left(60^{\circ}, 20^{\circ}\right),\left(60^{\circ}, 30^{\circ}\right)$, $\left(60^{\circ}, 45^{\circ}\right)$. These choices are motivated by the need to generate strong separated flow during downstroke and furthermore cover the typical values observed in nature.

The translating and rotating velocities in regions $R$ follow fourth order polynomial motion laws, ensuring their continuity throughout the flapping period. The translating velocity $V$ and the angle of attack $\alpha$ are, respectively 0 and $\left(180+\alpha_{\mathrm{d}}-\alpha_{\mathrm{u}}\right) / 2\left(^{\circ}\right)$ at the end of a stroke. Their evolutions during a flapping period $T$ are displayed in Fig. 2. Note that the period $T$, starting at the beginning of upstroke, is calculated as:

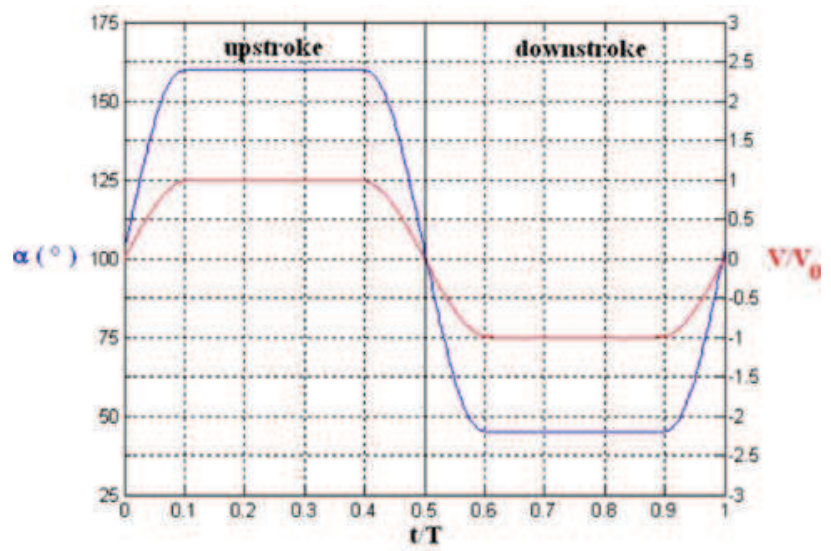

Fig. 2 Temporal evolutions of the angle of attack and translating velocity $\left(\alpha_{\mathrm{d}}=45^{\circ}, \alpha_{\mathrm{u}}=20^{\circ}\right)$ leading to a flapping frequency $f$ of approximately $10 \mathrm{~Hz}$ in real airflow configurations (with $c=10 \mathrm{~mm}$ ). Dynamical scaling using the Reynolds similarity brings $f=0.02 \mathrm{~Hz}$ for the experimental water flow configurations described in the following section (with $c=60 \mathrm{~mm}$ ).

\subsection{Experimental setup}

The experiments are conducted in a $1 \times 1 \times 2-\mathrm{m}^{3}$ water tank made of altuglas. A NACA0012 profile of chord $60 \mathrm{~mm}$ and span $50 \mathrm{~cm}$ placed in the water tank translates and rotates according to programmed motion laws. The translational and rotational motions are driven separately through the use of two servo-controlled motors. Their respective mechanical transmissions are achieved by means of an endless screw and pulleys as shown in Fig. 3a. The airfoil is connected at both ends to Plexiglas plates which limit three-dimensional effects, i.e. spanwise flow.

Two JAI 8-bits cameras are placed side by side, focusing on the spanwise symmetry plane of the airfoil. The resolution of the sensors is $1,600 \times 1,200$ pixels with a pixel size of $7.4 \times 7.4 \mu \mathrm{m}$. Each camera is equipped with a 50 $\mathrm{mm}$ focal length lens, $F_{\#} 2.8$, for a $370 \times 280-\mathrm{mm}^{2}$ area imaging. A continuous argon laser system (Argon SpectraPhysics of $10 \mathrm{~W}$ maximum power) is used to provide a 4.5W laser. $30 \%$ of the laser is guided through spherical and cylindrical lenses, illuminating one side of the airfoil; $70 \%$ is transported by means of an optical fiber to illuminate the opposite side. This method is adopted to limit the shadow effects (Fig. 3). Hollow silver coated glass particles with a mean diameter of $15 \mu \mathrm{m}$ are used for the seeding. The concentration is defined as $C=1.35 \times 10^{9}$ (particles $/ \mathrm{m}^{3}$ ) in order to ensure a proper number of particles per PIV correlation interrogation window (typically between 10 and 20).

TR-PIV is performed by taking images every $1 / 1,000$ period. The time step $(\Delta t \approx 50 \mathrm{~ms})$ allows an accurate calculation of both velocity and acceleration flow fields. 

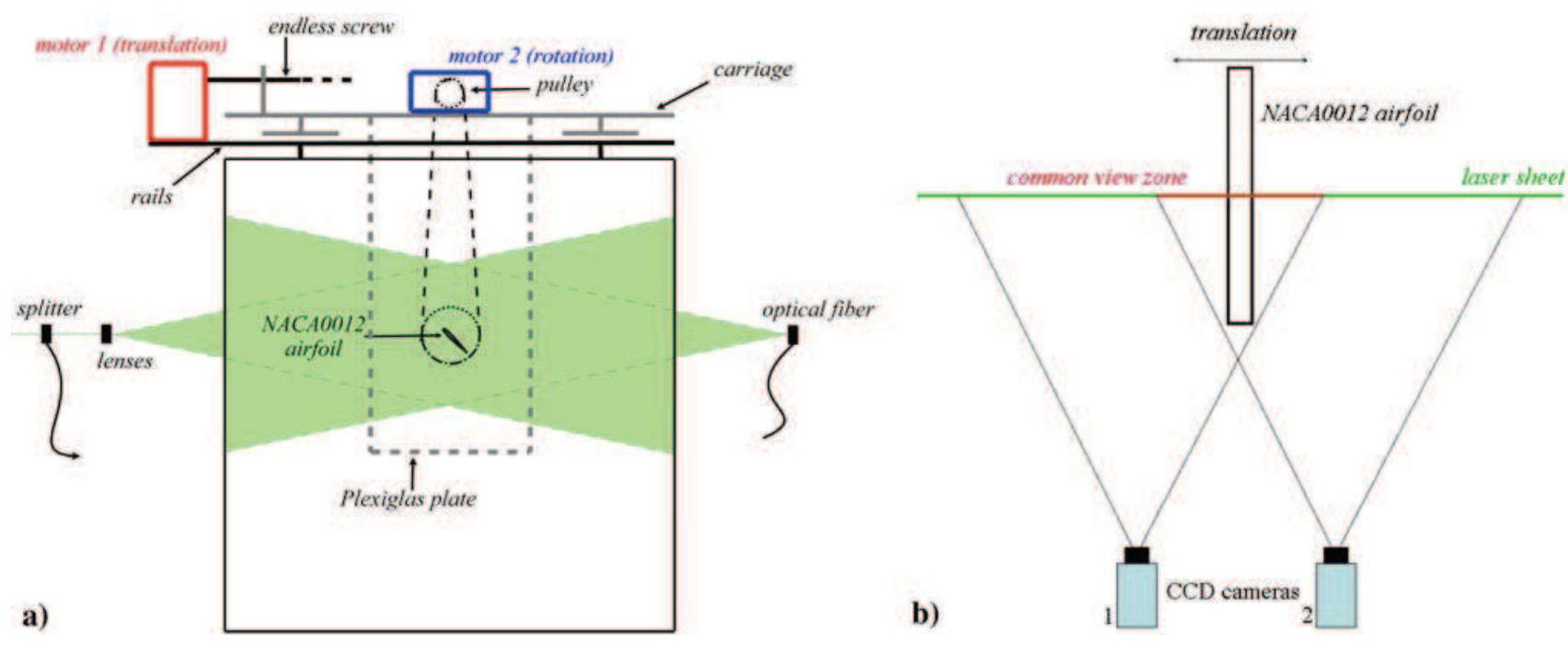

Fig. 3 Experimental setup: front view (a) upside view (b)

The acquisition is synchronised with the servo-controlled motor variators which deliver a tension signal at the beginning of the seventh period of the flapping motion for which the flow is ensured to be periodical.

\subsection{Post-processing}

The two-dimensional velocity flow fields for each camera are deduced from the TR-PIV images using the 7.2 LaVision software. A multipass algorithm with a final interrogation window size of $16 \times 16$ pixels and $50 \%$ overlapping is applied. Image deformation and round pyramidal weighting function are used. Spurious velocities are identified and replaced using both peak ratio and median filters. The average percentage of spurious vectors calculated at each instant over the whole flapping cycle (1,000 instants) is roughly $2 \%$. The final velocity flow fields are reconstructed using kriging interpolation from the combination of both camera information and known boundary conditions on the airfoil surface (Fig. 3b). The advantages of using two cameras are (1) to increase the spatial resolution and (2) to avoid inaccessible regions due to the perspective effect. The cameras share a common view zone so that the velocity flow fields on the left and right side of the profile come from the left and right cameras, respectively. The final flow fields have an area of $570 \times 280 \mathrm{~mm}^{2}$.

\subsection{Investigation methods}

The flow topology is analyzed by means of the adimensional vorticity flow fields computed from the basis of TRPIV velocity flow fields as shown in Eq. 3 for a twodimensional flow. The vorticity criterion is here convenient since the flow is assumed to be generally laminar, exhibiting distinguished shear layers and vortical structures (Jeong and Hussain 1995).

$\omega_{z}^{*}=\frac{c}{V_{0}}\left(\frac{\partial u}{\partial y}-\frac{\partial v}{\partial x}\right)$

The spatial and temporal behaviours of the vortical structures are correlated with the unsteady lift and drag determined by means of the momentum equation approach. The latter relies on the integration of flow variables inside and around a control volume surrounding the airfoil (Noca et al. 1997, 1999; Unal et al. 1997) (Fig. 4). Equation 4 gives the instantaneous force $\vec{F}(t)$ experienced by the airfoil in function of four components:

$$
\begin{aligned}
\vec{F}(t)= & -\rho \iiint_{V} \frac{\partial \vec{V}}{\partial t} \mathrm{~d} V-\rho \iint_{S}(\vec{V} \cdot \vec{n})\left(\vec{V}-\vec{V}_{S}\right) \mathrm{d} S \\
& -\iint_{S} P \vec{n} \mathrm{~d} S+\iint_{S} \bar{\tau} \vec{n} \mathrm{~d} S
\end{aligned}
$$

where $\vec{n}$ is the normal to the control surface $S$ limiting the control volume $V, \rho$ the fluid density, $\vec{V}$ the flow velocity

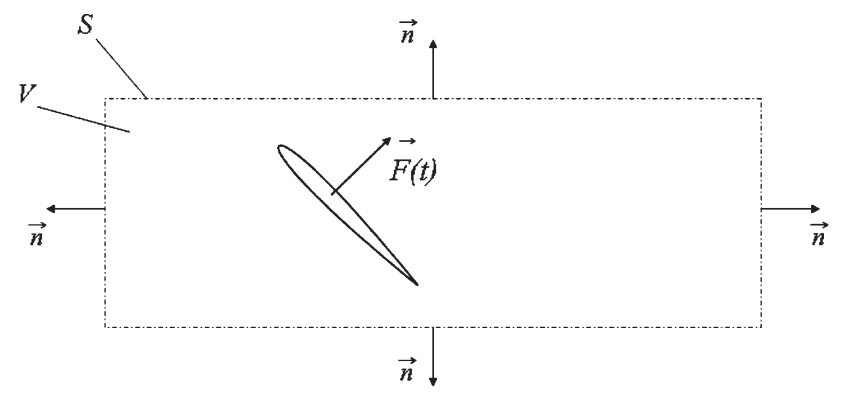

Fig. 4 Control volume definition 
vector, $\vec{V}_{S}$ the velocity of the control volume and $\overline{\bar{\tau}}$ the viscous stress tensor. The unsteady and convective terms (the first two right hand side contributions) are directly deduced from the TR-PIV velocity flow fields and account for the rate of change of momentum due to the flow unsteadiness within the control volume and the convection across the control surface, respectively. Note that the convective term is not integrated over the airfoil surface since equals zero for a no through flow boundary condition. The third term represents the normal stresses acting on the control surface. Its deduction requires the knowledge of the pressure $p$, obtained through the integration of the pressure gradient along the control surface. The pressure gradient is calculated from the momentum equation:

$\frac{\mathrm{D} \vec{V}}{\mathrm{D} t}=-\frac{1}{\rho} \nabla p+v \Delta \vec{V}$.

The last term accounts for the viscous stresses on the control surface. It is derived from the velocity flow fields, but may be neglected for preponderant pressure force flows or if the control surface is sufficiently far away from the airfoil.

The momentum equation approach is particularly appealing since it is non-intrusive and allows an accurate correlation between flow behaviour and force mechanisms, which is not a priori the case when separate techniques are used. Furthermore, the method is convenient for measuring forces at low Reynolds numbers and on moving bodies. For such cases, the use of piezoelectric gauges introduces non-negligible relative errors caused by the range of measures (e.g. 10-g loads) as well as the presence of an inertial component (for non-constant motion), respectively. Details concerning the numerical solving methods of Eq. 4 applied on a flapping flight configuration may be found in Jardin et al. (2008). Nevertheless, we may precise that the pressure term stands as the critical point since the pressure integration by means of Eq. 5 is subjected to both (1) error emphasizing due to the presence of differential operators and (2) error propagation phenomenon. Besides, as a result to the position of the integration limits, the contribution of this pressure term is enhanced in the case of drag prediction comparatively to lift prediction, involving different level of accuracy for both components. What's more, one should expect the pressure contribution; hence, the result accuracy, to be specifically strong in large wake configurations. Previous tests on numerical data subjected to $\pm 10 \%$ random noise on the velocity vectors revealed a mean error on the unsteady, convective and pressure contributions of $2,3.5,11 \%$ and $0.5,0.5,6 \%$ for the drag and lift predictions, respectively (David et al. 2009).

\subsection{Aerodynamic indicators}

The unsteady aerodynamic force $\vec{F}(t)$ is decomposed into the lift and drag components $F_{1}$ and $F_{\mathrm{d}}$, respectively perpendicular and collinear to the stroke plane as shown in Fig. 1. In the specific cases of symmetric flapping motions, the mean drag magnitude generated over a stroke is quasiidentical whether the airfoil is going downstroke or upstroke. Thus, setting the stroke plane as the horizontal axis brings $F_{x}= \pm F_{\mathrm{d}}$ and $F_{y}=F_{1}$ ensuring the hovering flight condition (i.e. zero mean horizontal force). In this paper, we focus on asymmetric flapping motions, the asymmetry being introduced through non-equal downstroke and upstroke angles of attack. For such cases, the mean drag magnitude over a stroke is different whether the airfoil is going downstroke or upstroke, such that the stroke plane should be tilted to maintain hovering. The vertical and horizontal aerodynamic forces are hence a combination of both lift and drag, depending on the stroke plane incidence $\beta$ (Eq. 6):

$$
\begin{aligned}
& F_{x}= \pm F_{\mathrm{d}} \cos (\beta)-F_{1} \sin (\beta) \\
& F_{y}=F_{1} \cos (\beta) \pm F_{\mathrm{d}} \sin (\beta)
\end{aligned}
$$

( + or - signs, respectively used for downstroke or upstroke), $\beta$ being computed for prescribed motion laws to satisfy the hovering flight condition:

$$
\int_{0}^{T} F_{x}(t) \mathrm{d} t=0
$$

Consequently, the common definition of the efficiency ratio $F_{1} / F_{\mathrm{d}}$ loses its significance since $F_{1}$ no longer represents the effective lifting force. A more suitable indicator of the aerodynamic efficiency is considered as the ratio $F_{y} / F_{\mathrm{d}}$. This definition a priori suggests enhanced efficiency ratios, the harmful influence of $F_{\mathrm{d}}$ being weakened through its contribution to $F_{y}$.

The force components $F_{x}, F_{y}, F_{\mathrm{d}}, F_{1}$ are adimensionalized using the downstroke and upstroke translating velocity $V_{0}$ and the chord of the airfoil $c$. The aerodynamic coefficients are defined as:

$$
C_{i}=\frac{2 F_{i}}{\rho c V_{0}^{2}} .
$$

\section{Results and discussion}

The aim of this section is to compare the unsteady loads resulting from different asymmetric flapping motions and to provide physical explanations by correlating them to the corresponding time dependent vorticity flow fields. In view of flapping wing concept application to MAVs, the 
Fig. 5 Adimensional (bottom) vorticity flow fields at $t / T=0.5($ left $)$ and $t / T=0.75$ (right) experimental (top) and DNS

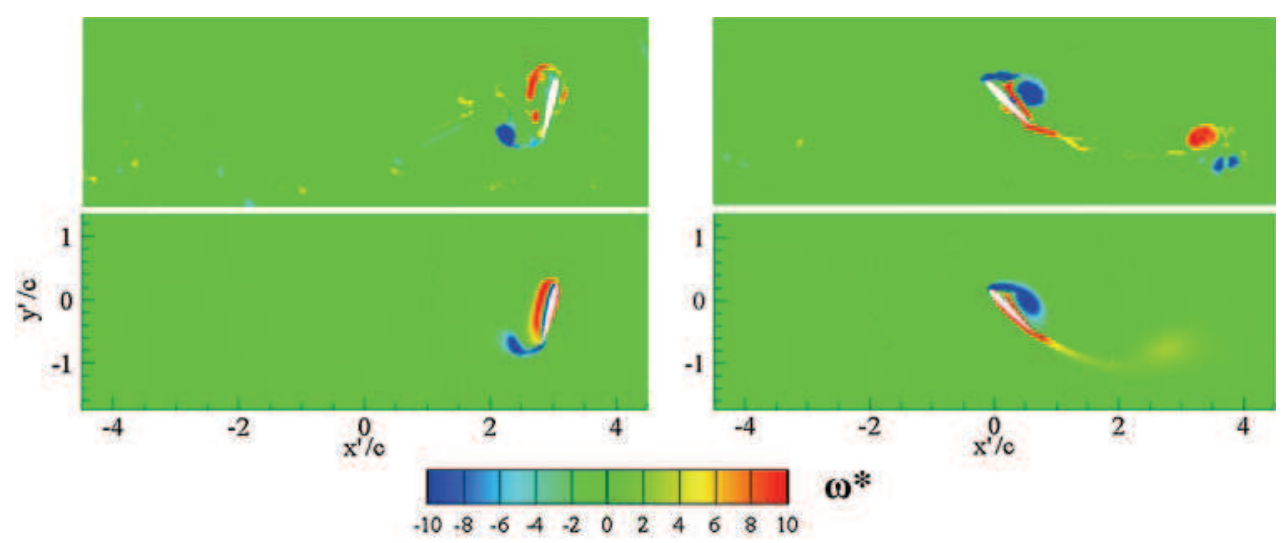

aerodynamic performances (e.g. lifting force, efficiency ratio) of such non-biological simplified motions are calculated and analysed. A brief confrontation with direct numerical simulation (DNS) results is first performed for both validating the momentum equation approach and demonstrate continuity with the previous complementary work of Kurtulus et al. (2008).

\subsection{Experimental versus numerical results}

For sake of conciseness, the comparison between experimental and numerical results is limited to the downstroke part of the asymmetric configuration $\alpha_{d}=45^{\circ}, \alpha_{u}=20^{\circ}$. The numerical loads and vorticity flow fields are obtained by directly solving the two-dimensional Navier-Stokes equations using a moving mesh flow solver (Kurtulus et al. 2005). Figure 5 displays the experimental and numerical adimensional vorticity flow fields at stroke reversal $(t /$ $T=0.5)$ and in the middle of downstroke $(t / T=0.75)$. Figure 6 shows the temporal evolution of the corresponding lift and drag coefficients during the totality of downstroke.

The comparison shows good agreement between the experimental and numerical flow topologies, both in the existence of the main vortical structures and in their vorticity levels. However, the numerical solver demonstrates difficulties predicting their exact location and temporal evolution (e.g. shedding); and hence, exhibits slight discrepancies with the time-dependent experimental aerodynamic lift. We can observe from Fig. 6 that the experimental and numerical lifts match until the shedding of the leading edge vortex (represented as a negative vorticity region on the extrados at time $t / T=0.75$, Fig. 5) which leads to a lower experimental lift at time $t / T=0.85$. The prediction of drag is more delicate both numerically and experimentally. Indeed, the flow solver is subjected to numerical diffusivity, as observed when looking at the vorticity levels of the starting vortex at $t / T=0.75$ (Fig. 5), which may tend to underestimate the drag. Furthermore,

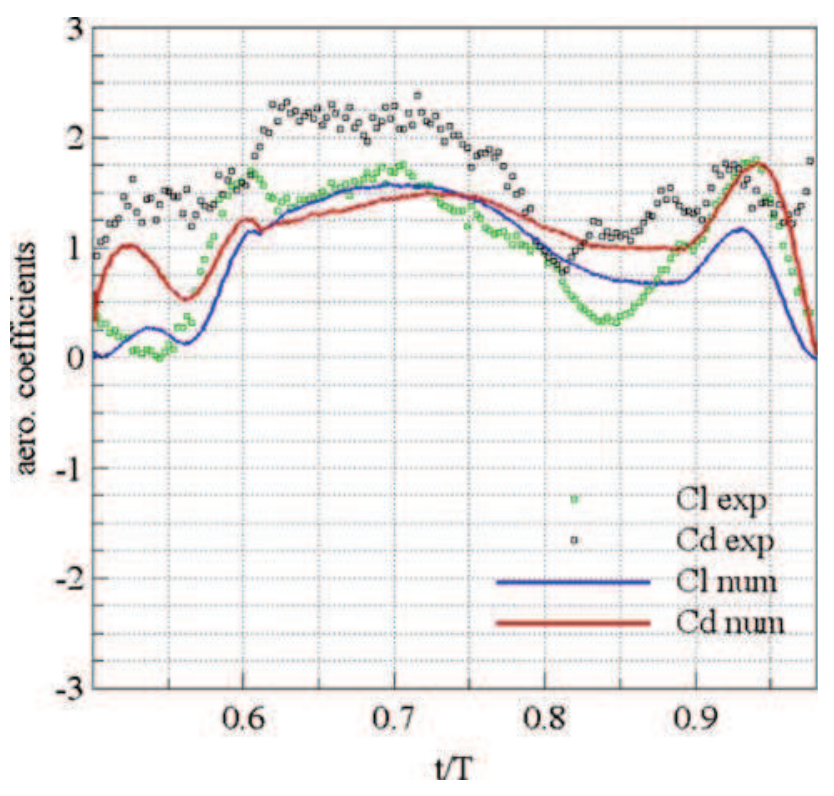

Fig. 6 Temporal evolution of the experimental and DNS downstroke aerodynamic coefficients $C_{1}$ and $C_{\mathrm{d}}$

the deduction of the experimental drag imposes to calculate the pressure in the wake, introducing non-negligible errors (Jardin et al. 2008). Thus, the comparison between numerical and experimental drags shows an offset between characteristic levels but demonstrates comparable temporal tendencies. Besides, the interesting point arising from this comparison is that similar experimental and numerical vortical flow fields lead to comparable experimental and numerical aerodynamic loads. Also, if the drag prediction may be subjected to quantitative uncertainties, the present parametrical study may accurately rely on qualitative comparisons.

\subsection{Wake topology}

The downstroke and upstroke angles of attack are chosen as parameters. The parametrical study is conducted fixing 

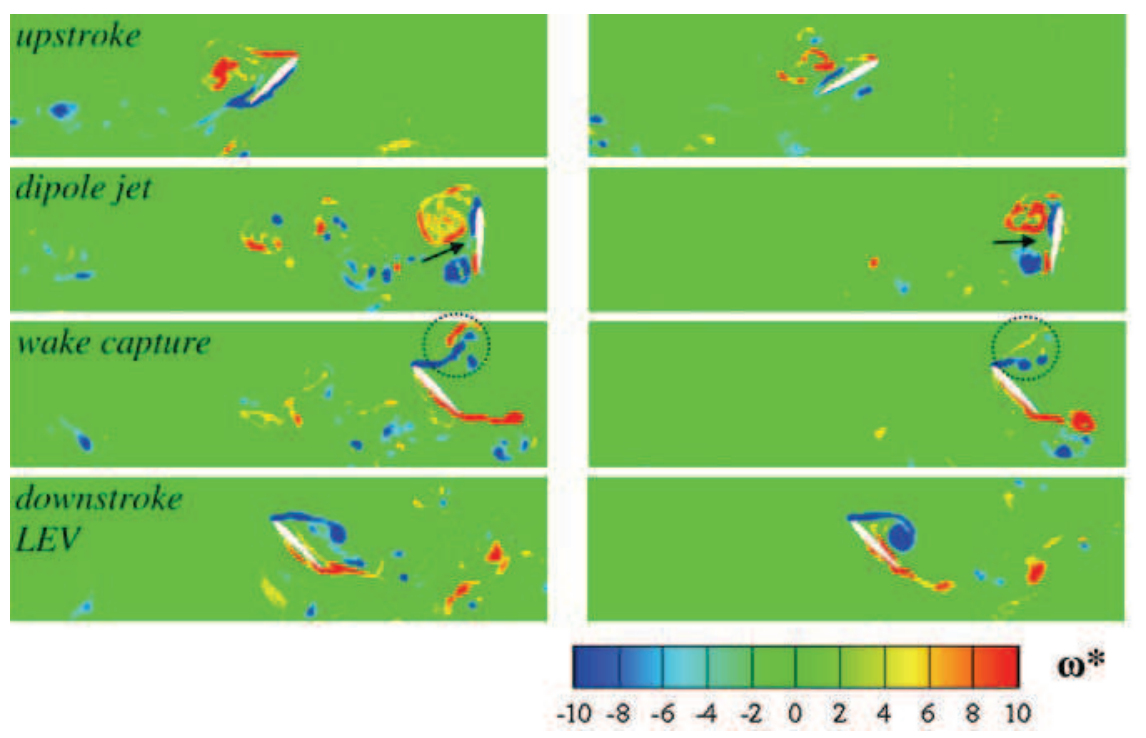

Fig. 7 Adimensional vorticity flow fields at times $t / T=0.25,0.5,0.6,0.75$ (top to bottom) for the configurations $\alpha_{\mathrm{d}}=45^{\circ}, \alpha_{\mathrm{u}}=45^{\circ}, 30^{\circ}, 20^{\circ}$ (left to right)

the downstroke angle of attack to either $30^{\circ}, 45^{\circ}$ or $60^{\circ}$; hence, distinguishing three groups. For each group, three values of the upstroke angle of attack are tested, leading to a total of nine configurations. The configurations of a similar group have quasi identical downstroke kinematics, thus differing quasi exclusively on their upstroke kinematics. As a consequence, inside a specific group, the comparison of the vortex dynamics observed during the downstroke of the different configurations directly reveals the influence of the wake capture phenomenon. The main analysis is carried out on the $\alpha_{d}=45^{\circ}$ group, for which the normal hovering configuration $\left(\alpha_{\mathrm{u}}=45^{\circ}\right)$ is described in details in Kurtulus et al. (2008). Figure 7 displays the adimensional vorticity flow fields for the three $\alpha_{\mathrm{d}}=45^{\circ}$ configurations. Note that the stroke plane is represented horizontally to facilitate comparisons.

In the particular normal hovering configuration, the upstroke angle of attack is set to $45^{\circ}$, inducing a highly unsteady vortical wake at $t / T=0.25$. At the end of upstroke $(t / T=0.5)$, strong leading and trailing edge vortices (LEV and TEV) are attached to the airfoil, forming a dipole which generates a fluid jet oriented towards the intrados. This jet acts as a drag enhancer and contributes to lower the aerodynamic efficiency. As the airfoil rotates and initiates the downstroke translational phase, the LEV is captured, accelerating and strengthening the leading edge separation; hence, the formation of a new counter rotating downstroke LEV $(t / T=0.6)$. The latter is carried away from the airfoil under the action of the upstroke LEV without being instantaneously shed $(t / T=0.75)$. Its consequent relative position to the airfoil extrados implies relatively weak aerodynamic forces.
Asymmetry is introduced by lowering the upstroke angle of attack, while keeping the downstroke angle of attack fixed to $45^{\circ}$. In the first asymmetric case, $\alpha_{u}$ is set to $30^{\circ}$, leading, as in the normal hovering configuration, to a strong separated flow during the upstroke translational phase. However, relatively to the normal hovering configuration, the dimensions and strengths of the vortices are here less pronounced. At stroke reversal, the vortex dipole is hence likely to produce a weaker fluid jet whose influence on aerodynamic efficiency is less penalizing though since it partially contributes to the generation of vertical aerodynamic force (the stroke plane being inclined). Furthermore, a direct consequence of reduced vortex dimensions and strengths relies on the specificity of the wing/wake interaction. At time $t / T=0.6$, the influence of the upstroke LEV on the formation of a new downstroke LEV is lower than observed for the normal hovering case. Thus, we can remark that the downstroke LEV remains closer to the airfoil surface, presupposing enhanced lift and drag $(t / T=0.75)$. The asymmetry is pushed further in the second asymmetric case where $\alpha_{u}$ is set to $20^{\circ}$. In this particular configuration, the upstroke is dominated by an attached flow between time $t / T=0.2$ and time $t / T=0.35$, besides the fact that $\alpha_{u}$ is above the critical stall incidence for a NACA0012 profile. This attachment may be explained by the presence of a fluid downwash, resulting from the lift generation of the previous strokes, and which tends to decrease the effective angle of attack. The influence of the fluid downwash is less perceptive at the end of the strokes such that a separation still lately occurs. The growth of the resulting upstroke LEV is limited, leading to a weak vortex dipole at stroke reversal. In contrast to the 

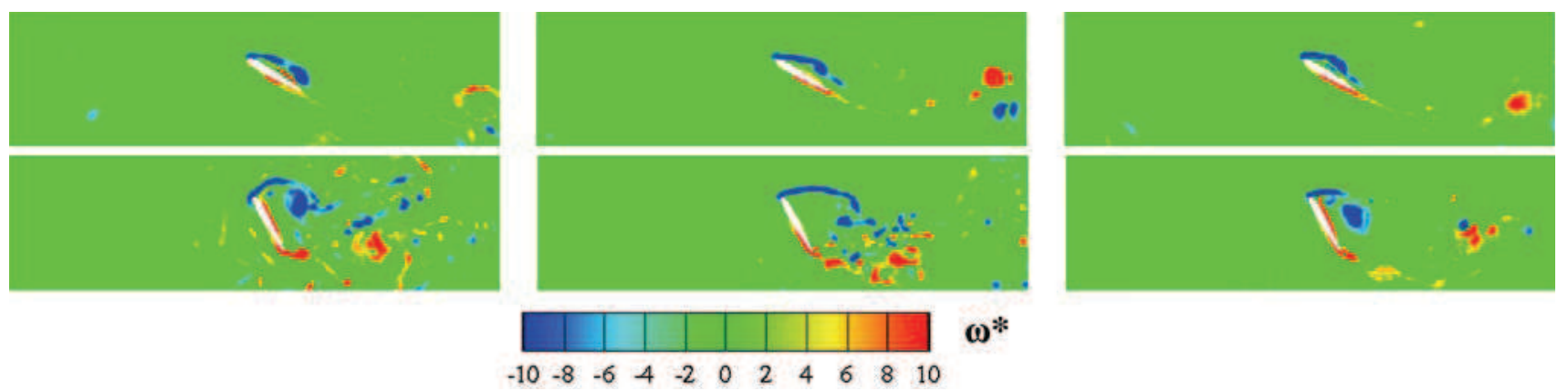

$\begin{array}{lllllllllll}-10 & -8 & -6 & 4 & -2 & 0 & 2 & 4 & 6 & 8 & 10\end{array}$

Fig. 8 Adimensional vorticity flow fields at times $t / T=0.75$ for the configurations $\alpha_{\mathrm{d}}=30^{\circ}$ (top), $\alpha_{\mathrm{u}}=30^{\circ}, 20^{\circ}, 10^{\circ}$ (left to right) and $\alpha_{\mathrm{d}}=60^{\circ}$ (bottom), $\alpha_{\mathrm{u}}=45^{\circ}, 30^{\circ}, 20^{\circ}$ (left to right)

previous configurations, as the wing initiates the downstroke phase, the upstroke LEV slides under the intrados instead of being pushed above the leading edge. Therefore, the upstroke LEV has no influence on the formation of the downstroke LEV which develops smoothly on the extrados, forming a high lift generating low pressure region.

Figure 8 shows the adimensional vorticity flow fields at time $t / T=0.75$ for the $\alpha_{\mathrm{d}}=30^{\circ}$ and $\alpha_{\mathrm{d}}=60^{\circ}$ groups. When $\alpha_{\mathrm{d}}=30^{\circ}, \alpha_{\mathrm{u}}$ is successively fixed to $30^{\circ}, 20^{\circ}$ and $10^{\circ}$. In the normal hovering case $\left(30^{\circ}, 30^{\circ}\right)$, the position of the upstroke LEV is such that it slides under the intrados as the wing initiates the downstroke phase. On the contrary, in the first asymmetric case $\left(30^{\circ}, 20^{\circ}\right)$, the upstroke LEV is split into two smaller structures, one being ejected over the leading edge, hence slightly affecting the formation of the downstroke LEV, the other sliding under the intrados. In the last asymmetric case $\left(30^{\circ}, 10^{\circ}\right)$, because of the weak value of the upstroke angle of attack, the upstroke wake exhibits closely attached structures which slide under the intrados at stroke reversal. Consequently, it is denoted from Fig. 8 that the downstroke LEV is closer to the airfoil surface in the $\left(30^{\circ}, 30^{\circ}\right)$ and $\left(30^{\circ}, 10^{\circ}\right)$ configurations than in the $\left(30^{\circ}, 20^{\circ}\right)$ one, giving additional evidences of the wake capture phenomenon. Nevertheless, despite the absence of significant wake capture in both cases, the $\left(30^{\circ}\right.$, $\left.30^{\circ}\right)$ and $\left(30^{\circ}, 10^{\circ}\right)$ downstroke LEVs are not identical. This difference may arise from different rotation speed or more likely from different downwash intensity. When $\alpha_{\mathrm{d}}=60^{\circ}$, the corresponding cases demonstrate more complex behaviours. The upstroke wake of the $\left(60^{\circ}, 45^{\circ}\right)$ configuration is highly unsteady and vortical resulting in a severe wing/wake interaction at the beginning of upstroke. A first downstroke LEV is hence rapidly formed and shed, leaving room to a second downstroke LEV which still tends to detach from the extrados at time $t / T=0.75$. In the $\left(60^{\circ}, 30^{\circ}\right)$ configuration, the wake capture is less pronounced such that the first downstroke LEV is not promptly shed, leading to a highly stretched second downstroke LEV. In the last $\left(60^{\circ}, 20^{\circ}\right)$ configuration, the upstroke LEV sweeps under the intrados as previously observed for low upstroke angles of attack. The downstroke LEV is thus not influenced by the previous wake.

\subsection{Aerodynamic performances}

The analysis of the vortical flow fields revealed that the desymmetrization of flapping motions reduces the effect of wake capture; hence, leading in most cases to a closely attached downstroke LEV. Consequently, the latter being assimilated to a low pressure suction region, the generation of aerodynamic loads should be enhanced during downstroke comparatively to normal hovering flapping flight configurations. Figure 9 confronts the temporal evolution of the downstroke lift component of each experimented configuration belonging to a similar $\alpha_{d}$ group. We remind that within a group, the downstroke kinematics of each configuration are roughly identical, resulting in equivalent quasi-steady forces. Notwithstanding this similarity, Fig. 9 denotes significantly different aerodynamic lift coefficients during the downstroke pure translational phase $(t / T[0.61$; 0.89]), consistent with the previous remarks on the attachment of the downstroke LEV. Precisely, it is shown that the highest downstroke lifts are attained for smaller upstroke angles of attack (green squares). Furthermore, noticeable different tendencies at the beginning of downstroke are exposed, supporting the evidence of dissimilar wing/wake interactions. In particular, at $t / T=0.55$, the upstroke LEV is pushed over the leading edge for the configurations $\left(45^{\circ}, 45^{\circ}\right)$ and $\left(45^{\circ}, 30^{\circ}\right)$ resulting in a rapid augmentation of the lift coefficient which reaches approximately 1.5. Notice that this lift bump has been put into evidence by Birch and Dickinson (2003) and similarly attributed to the wake capture phenomenon. On the contrary, the upstroke LEV slides under the intrados for the configuration $\left(45^{\circ}, 20^{\circ}\right)$, inhibiting the lift deriving from the wing acceleration $(\mathrm{Cl}=0$ at $t / T \approx 0.55)$. Finally, the additional circulation provided by the combined translating and rotating motions (Kramer effect) implies the presence 

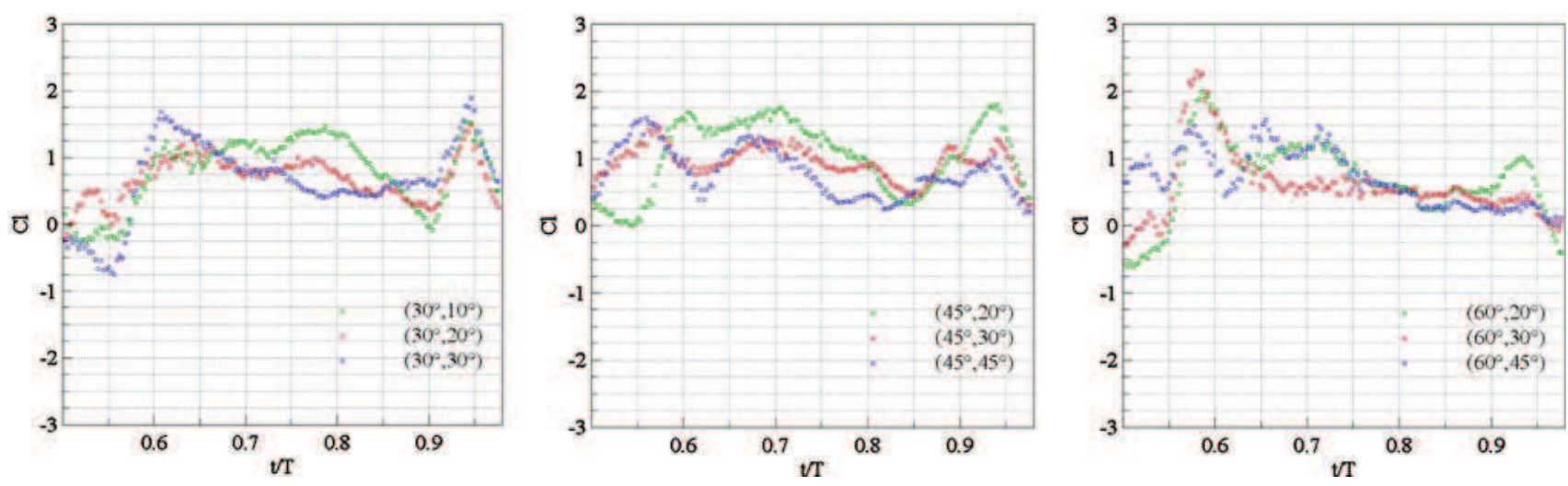

Fig. 9 Time-dependent downstroke lift coefficients of the nine configurations grouped according to $\alpha_{\mathrm{d}}$ values $\left(\alpha_{\mathrm{d}}=30^{\circ}, 45^{\circ}\right.$ and $60^{\circ}$ from left to right)

of a second lift bump at the end of downstroke as measured by Sane and Dickinson (2002). This bump is less perceptive when the difference between $\alpha_{\mathrm{d}}$ and $\alpha_{\mathrm{u}}$ is low (e.g. $\alpha_{\mathrm{d}}=60^{\circ}$ configurations), i.e. weakened rotation speed. Consequent to these remarks, the downstroke time-averaged lift coefficients are, respectively $0.78,0.92$ and 1.02 , for the configurations $\left(45^{\circ}, 45^{\circ}\right),\left(45^{\circ}, 30^{\circ}\right)$ and $\left(45^{\circ}, 20^{\circ}\right)$, proving the benefit of desymmetrization. It is not clear whether the asymmetric configurations numerically studied by Wang $(2000,2004)$ do benefit from such vortex dynamics (more or less significant wake capture) or not.

Despite the influence of the vortex dynamics on the production of aerodynamic forces, it is of interest to highlight the influence of tilting the stroke plane on the contributions of the lift and drag components to the resulting vertical force; hence, the efficiency ratio. Indeed, as previously expressed, the introduction of asymmetry implies an inclined stroke plane in order to verify the hovering flight condition (Eq. 7). For the $\alpha_{\mathrm{d}}=45^{\circ}$ group, the calculation of the mean lift and drag coefficients leads to $\beta=-2^{\circ}, 23^{\circ}$ and $38^{\circ}$ for the configurations $\left(45^{\circ}, 45^{\circ}\right)$, $\left(45^{\circ}, 30^{\circ}\right)$ and $\left(45^{\circ}, 20^{\circ}\right)$. The respective resulting mean vertical force coefficients (averaged over a flapping period T) are obtained by means of Eq. 6: $\overline{C_{y}}=0.85,1.07$ and 1.23. These results suggest that despite the harmful influence of the upstroke phase, for which the drag component tends to prevent the wing from keeping aloft, asymmetric motions generate enhanced vertical aerodynamic force. Relative to the normal hovering configuration, the increase reaches $45 \%$. Nevertheless, one should keep in mind the difficulties linked to the experimental determination of drag through the momentum equation approach (Jardin et al. 2008), such that presented levels are likely to be slightly overestimated. Finally, the mean efficiency ratios are found to be improved: $\overline{C_{\text {er }}}=0.53,0.82$ and 1.02. The influence of desymmetrization on aerodynamic performances is roughly identical for the $\alpha_{\mathrm{d}}=30^{\circ}$ group, the efficiency ratio coefficients being $0.90,0.94$ and 1.42 for the respective configurations $\left(30^{\circ}, 30^{\circ}\right),\left(30^{\circ}, 20^{\circ}\right)$ and $\left(30^{\circ}, 10^{\circ}\right)$. However, as previously expressed, the enhanced value of the mean downstroke lift of the asymmetric configuration $\left(30^{\circ}, 10^{\circ}\right)$ compared to the normal configuration $\left(30^{\circ}, 30^{\circ}\right)$ (respectively 0.78 and 0.67 ) does not arise from the absence of significant wake capture, but more likely from a weaker fluid downwash which may confine the LEV closer to the extrados. The configurations of the $\alpha_{\mathrm{d}}=60^{\circ}$ group are characterized by large messy downstroke wakes propitious to errors on the pressure determination while predicting the loads. Precisely, the influence of specific parameters such as the dimensions and position of the control volume is amplified comparatively to the other $\alpha_{d}$ groups, which may lead to $40 \%$ discrepancies on the instantaneous values when wing/wake interactions occur. What's more, the complex fluid dynamics exhibited in such cases make the interpretation and the comparison of the resulting time-dependent coefficients particularly delicate. Nevertheless, the mean efficiency ratios obtained for the $\left(60^{\circ}, 45^{\circ}\right),\left(60^{\circ}, 30^{\circ}\right)$ and $\left(60^{\circ}, 20^{\circ}\right)$ (respectively 0.49 , 0.81 and 1.31) once again corroborate the previous results, suggesting the beneficial influence of desymmetrization. As a conclusion, despite the "not so crucial" importance of the influence of the wake capture on the attachment of the downstroke LEV (the flow being strongly separated in all cases), it is probable that asymmetric configurations highly benefit from the contribution of drag to vertical force.

The previous calculations permit the representation of instantaneous aerodynamic force vectors during the flapping motions as displayed in Fig. 10 for the $\alpha_{\mathrm{d}}=45^{\circ}$ group. Such representation puts into evidence the phenomenon of wake capture as well as the Kramer effect through the presence of strong vectors, respectively at the beginning and at the end of a stroke. As previously expressed, these mechanisms are also denoted by bumps in the temporal evolution of the lift coefficients (Fig. 9). Furthermore, it 

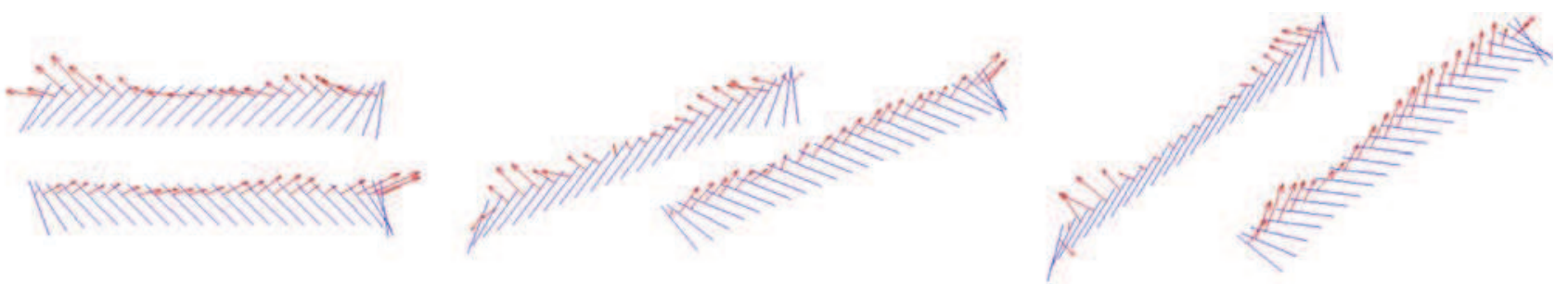

Fig. 10 Instantaneous aerodynamic force vectors for the configurations $\left(45^{\circ}, 45^{\circ}\right),\left(45^{\circ}, 30^{\circ}\right)$ and $\left(45^{\circ}, 20^{\circ}\right)$ (left to right)

clearly appears that the $\left(45^{\circ}, 20^{\circ}\right)$ configuration fully benefits from the contribution of drag, the force vectors being nearly vertical at the beginning of downstroke.

\section{Conclusion}

Flapping wings recently appeared to be a high aerodynamic performance lifting device at low Reynolds numbers. In order to consider such concept as a possible alternative to the conventional fixed and rotary wings for the development of MAVs, it is of interest to investigate nonbiological wing profiles and kinematics. The understanding of the influence of the latter on the aerodynamic performances of flapping wings may lead to lift/efficiency enhancement. In this paper, an experimental parametrical study of simplified two-dimensional asymmetric hovering flapping motions was carried out using 2D/2C TR-PIV measurements as a basis to evaluate both time-dependent vorticity flow fields and unsteady aerodynamic loads. Relative to normal hovering configurations, asymmetry was introduced by differentiating the downstroke and upstroke angles of attack, chosen as parameters. It is demonstrated that lowering the upstroke angle of attack while keeping a fixed downstroke angle of attack results in a reduced upstroke wake whose influence on the formation of the downstroke leading edge vortex (through the wake capture phenomenon) is hence significantly weakened. Furthermore, in some specific cases, the wake capture phenomenon no longer affects the formation of the leading edge vortex which thus develops smoothly, closely attached to the extrados. The leading edge vortex being assimilated to a low pressure suction region, such characteristics (i.e. vortex position, strengths and dimensions) lead to enhanced downstroke lift and drag. Besides, through the hovering flight condition, desymmetrization leads to an inclined stroke plane which implies that both lift and drag contribute to the production of a lifting force. Consequent to these fluid dynamics and flight mechanics considerations, asymmetric hovering flapping motions are found to be specifically efficient in comparison to normal hovering flapping motions. Such remarks support the numerical work of Wang (2004) who proposed efficient asymmetric configurations. From this point, it is of interest to introduce other parameters to asymmetric configurations in order to further increase aerodynamic performances. As an example, the augmentation of the upstroke velocity is suggested, hence minimizing the harmful influence of upstroke relative to downstroke.

\section{References}

Bennett L (1970) Insect flight: lift and the rate of change of incidence. Science 167:177-179

Birch JM, Dickinson MH (2003) The influence of wing-wake interactions on the production of aerodynamic forces in flapping flight. J Exp Biol 206:2257-2272

David L, Jardin T, Farcy A (2009) Validation of unsteady drag and lift evaluation by TR-PIV. Meas Sci Technol (in revision)

Dickinson MH (1994) The effects of wing rotation on unsteady aerodynamic performance at low Reynolds number. J Exp Biol 192:179-206

Dickinson MH, Götz KG (1993) Unsteady aerodynamic performance of model wings at low Reynolds numbers. J Exp Biol 174:45-64

Dickinson MH, Lehmann FO, Sane SP (1999) Wing rotation and the aerodynamic basis of insect flight. Science 284:1954-1960

Ellington CP (1984) The aerodynamics of hovering insect flight I-V. Philos Trans R Soc Lond B 305:1-181

Gustafson KE, Leben R (1991) Computation of dragonfly aerodynamics. Comput Phys Commun 65:121

Jardin T, David L, Farcy A, Chatellier L (2008) Correlation between vortex structures and unsteady loads for flapping motion in hover. Fourteenth international symposium on applications of laser techniques to fluid mechanics, Lisbon

Jensen M (1956) Biology and physics of locust flight. III. The aerodynamics of locust flight. Philos Trans R Soc Lond B 239:511-552

Jeong J, Hussain F (1995) On the identification of a vortex. J Fluid Mech 285:69-94

Kramer M (1932) Die zunahme des maximalauftriebes von tragflugeln bei plotzlicher anstellwinkelvergrosserung. Z Flugtech Motorluftschiff 23:185-189

Kurtulus DF (2005) Numerical and experimental analysis of flapping motion in hover. Application to micro air-vehicles. PhD Thesis, Laboratoire d'Etudes Aérodynamiques, Université de Poitiers

Kurtulus DF, Farcy A, Alemdaroglu N (2005) Unsteady aerodynamics of flapping airfoil in hovering flight at low Reynolds numbers. AIAA 2005-1356, Reno

Kurtulus DF, David L, Farcy A, Alemdaroglu N (2008) Aerodynamic characteristics of flapping motion in hover. Exp Fluids 44:23-36

Liu H, Ellington CP, Kawachi K, van den Berg C, Willmott AP (1998) A computational fluid dynamic study of hawkmoth hovering. J Exp Biol 201:461-477 
Maxworthy T (1979) Experiments on the Weis-Fogh mechanism of lift generation by insects in hovering flight Part 1 . Dynamics of the 'fling'. J Fluid Mech 93:47-63

Noca F, Shiels D, Jeon D (1997) Measuring instantaneous fluid dynamic forces on bodies, using only velocity fields and their derivatives. J Fluids Struct 11:345-350

Noca F, Shiels D, Jeon D (1999) A comparison of methods for evaluating time-dependent fluid dynamic forces on bodies, using only velocity fields and their derivatives. J Fluids Struct 13:551578

Osborne MFM (1951) Aerodynamics of flapping flight with application to insects. J Exp Biol 28:221-245

Platzer MF, Jones KD (2006) Flapping wing aerodynamics-progress and challenges. AIAA 2006-500, Reno

Polhamus EC (1971) Predictions of vortex-lift characteristics by a leading-edge suction analogy. J Aircr 8:193-199

Sane SP, Dickinson MH (2001) The control of flight force by a flapping wing: lift and drag production. J Exp Biol 204:26072626

Sane SP, Dickinson MH (2002) The aerodynamic effects of wing rotation and a revised quasi-steady model of flapping flight. $\mathrm{J}$ Exp Biol 205:1087-1096

Singh B, Ramasamy M, Chopra I, Leishman J (2005) Insect-based flapping wings for micro hovering air vehicles: experimental investigations. American Helicopter Society International Specialists Meeting on Unmanned Rotorcraft, Phoenix

Somps C, Luttges M (1985) Dragonfly flight: novel uses of unsteady separated flows. Science 228:1326-1329

Sun M, Lan S (2004) A computational study of the aerodynamic forces and power requirements of dragonfly hovering. J Exp Biol 207:1887-1901

Sun M, Tang J (2002) Lift and power requirements of hovering flight in Drosophila virilise. J Exp Biol 205:2413-2427
Thomas ALR, Taylor GK, Srygley RB, Nudds RL, Bomphrey RJ (2004) Dragonfly flight: free-flight and tethered flow visualizations reveal a diverse array of unsteady lift-generating mechanisms, controlled primarily via angle of attack. J Exp Biol 207:4299-4323

Unal MF, Lin JC, Rockwell D (1997) Force prediction by PIV imaging: a momentum-based approach. J Fluids Struct 11:965971

van den Berg C, Ellington CP (1997) The three-dimensional leadingedge vortex of a hovering model hawkmoth. Philos Trans R Soc Lond B 352:329-340

von Holst E, Kuchemann D (1941) Biological and aerodynamical problems of animal flight. Naturwissenschaften 46:39-58

Walker GT (1925) The flapping flight of birds. J R Aeronaut Soc 29:590-594

Walker PB (1931) Growth of circulation about a wing and an apparatus for measuring fluid motion. ARC report

Wang ZJ (2000) Two-dimensional mechanism for insect hovering. Phys Rev Lett 85:2216-2219

Wang ZJ (2004) The role of drag in insect hovering. J Exp Biol 207:4147-4155

Wang ZJ, Birch JM, Dickinson MH (2004) Unsteady forces and flows in low Reynolds number hovering flight: two-dimensional computations vs robotic wing experiments. J Exp Biol 207:449-460

Weis-Fogh T (1973) Quick estimates of flight fitness in hovering animals, including novel mechanism for lift production. J Exp Biol 59:169-230

Weis-Fogh T, Jensen M (1956) Biology and physics of locust flight. I. Basic principles in insect flight. A critical review. Philos Trans $\mathrm{R}$ Soc B 239:415-458

Wu J, Sun M (2004) Unsteady aerodynamic forces of a flapping wing. J Exp Biol 207:1137-1150 